\title{
Universiteit
}

Leiden

The Netherlands

\section{Photon statistics from coupled quantum dots}

Gerardot, B.D.; Strauf, S.; Dood, M.J.A. de; Bychkov, A.; Badolato, A.; Hennessy, K.T.; ... ; Petroff, P.M.

\section{Citation}

Gerardot, B. D., Strauf, S., Dood, M. J. A. de, Bychkov, A., Badolato, A., Hennessy, K. T., ... Petroff, P. M. (2005). Photon statistics from coupled quantum dots. Physical Review Letters, 95(13), 137403. doi:10.1103/PhysRevLett.95.137403

Version: $\quad$ Not Applicable (or Unknown)

License: $\quad$ Leiden University Non-exclusive license

Downloaded from: https://hdl.handle.net/1887/64304

Note: To cite this publication please use the final published version (if applicable). 


\title{
Photon Statistics from Coupled Quantum Dots
}

\author{
Brian D. Gerardot, ${ }^{1}$ Stefan Strauf, ${ }^{1,2, *}$ Michiel J. A. de Dood, ${ }^{2}$ Andrey M. Bychkov, ${ }^{2, \dagger}$ Antonio Badolato, ${ }^{3}$ \\ Kevin Hennessy, ${ }^{3}$ Evelyn L. Hu, ${ }^{1,3}$ Dirk Bouwmeester, ${ }^{2}$ and Pierre M. Petroff ${ }^{1,3}$ \\ ${ }^{1}$ Materials Department, University of California Santa Barbara, Santa Barbara, California 93106, USA \\ ${ }^{2}$ Department of Physics, University of California Santa Barbara, Santa Barbara, California 93106, USA \\ ${ }^{3}$ ECE Department, University of California Santa Barbara, Santa Barbara, California 93106, USA
}

(Received 20 July 2004; published 23 September 2005)

\begin{abstract}
We present an optical study of two closely stacked self-assembled InAs/GaAs quantum dots. The energy spectrum and correlations between photons subsequently emitted from a single pair provide not only clear evidence of coupling between the quantum dots but also insight into the coupling mechanism. Our results are in agreement with recent theories predicting that tunneling is largely suppressed between nonidentical quantum dots and that the interaction is instead dominated by dipole-dipole coupling and phonon-assisted energy transfer processes.
\end{abstract}

PACS numbers: 78.67.Hc, 03.67.- a, 42.50.Dv, 78.55.Cr

Semiconductor quantum dots (QDs) are nanostructures that confine electrons and/or holes in all three dimensions. Excitons or single electron spins in QDs are promising candidates for the storage and manipulation of both classical and quantum bits [1]. Of particular interest for applications are semiconductor QDs in which excitons couple to photons. They display discrete energy levels [2], photon antibunching [3], and Rabi oscillations [4]. The coupling of two QDs has been proposed as a means to generate entangled photons and to realize quantum bit gate operations $[5,6]$. The quest for qubit operations in solid state has triggered several studies of the coupling processes in QD ensembles [7] and individual QD pairs [8-11]. Theoretical investigations predict that coupling between QDs can be caused by electron and/or hole tunneling $[9,12]$ or by dipole-dipole interaction of excitons [6,13-15]. Initial experiments reported large energy splittings up to $50 \mathrm{meV}$, attributed to tunnel coupling in identical QDs [9]. It is now understood that the dominant effect is the different size or strain situation of the individual QDs and that these splittings cannot be attributed to quantum mechanical coupling [10]. Refined theories that take into account the broken symmetry of nonidentical QDs predict repulsive forces between holes located on different dots, effectively preventing tunneling of excitons [16]. We present spectroscopic and photon-correlation measurements obtained from individual self-assembled InAs/GaAs QD pairs that demonstrate coupling between adjacent QDs and provide insight into the coupling mechanism and energy transfer between the QDs.

We chose to study QD pairs with a small vertical separation of $45 \AA$, where the coupling is expected to be pronounced. The InAs QDs were grown by molecular beam epitaxy on a (100) GaAs substrate via the StranskiKrastanow growth mode. Strain fields above each QD form nucleation centers for QDs in a second layer, leading to vertical QD stacking. The $s$-shell transitions of the QD layers are carefully tuned to nearly identical energies dur- ing the crystal growth $[17,18]$. Microphotoluminescence (PL) spectra were recorded using a $1.25 \mathrm{~m}$ spectrometer equipped with a charge coupled device. The QDs were nonresonantly excited at $780 \mathrm{~nm}$. A solid immersion lens on the sample surface was used to improve the photon collection efficiency.

The experimental scheme is introduced by first describing measurements on a single layer of uncoupled InAs QDs [19]. The PL spectrum in Fig. 1(a) shows the emission of a few QDs at $T=4 \mathrm{~K}$. The two main emission peaks correspond to the single exciton recombination of two individual QDs laterally separated by $\sim 2 \mu \mathrm{m}$. To determine whether or not these two QDs are coupled, the correlations between photons emitted from the QDs have been measured. The photons pass through a fiber beam splitter and $1 \mathrm{~nm}$ frequency filters in the two output modes ( $k$ and $l$ ) before reaching single photon detectors.
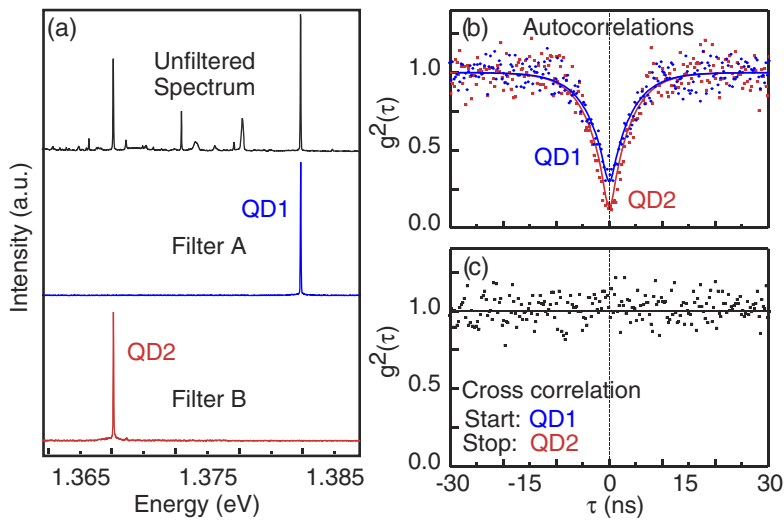

FIG. 1 (color online). (a) PL spectrum of a single layer of InAs/GaAs QDs. Interference filters were used to select two emission lines from two QDs, with lateral separation of $2 \mu \mathrm{m}$. (b) Photon autocorrelation for each line showing antibunching, $g^{(2)}(0)=0.31$ and 0.12 for QD1 and QD2, respectively. (c) Corresponding cross correlation showing $g^{(2)}(\tau)=1$ for all times, indicating that the lateral QDs are uncoupled. 
Measuring the difference in arrival time between photons at each of the two detectors provides a measurement of the second-order correlation function $g_{k l}^{(2)}(\tau)=\left\langle I_{k}(t) I_{l}(t+\tau)\right\rangle /$ $\left\langle I_{k}(t)\right\rangle\left\langle I_{l}(t)\right\rangle$, where $\left\langle I_{k}(t)\right\rangle$ is the expectation value of the intensity at time $t$. The autocorrelation function from a single QD is measured if both filters are tuned to the frequency of QD1 (or QD2). A single QD [3], just like a single atom, displays photon antibunching at $\tau=0$ [Fig. 1(b)]. For two identical but independent two-level emitters $g_{k=l}^{(2)}(0)=0.5$. If the two emitters are nonidenti$\mathrm{cal}$, as is the situation here, a postselection of the modes $k$ and $l$ can be made using different filters to measure a crosscorrelation function. In this case of uncoupled QDs, emission of a photon from QD1 at $\tau=0$ does not influence a photon emission event from QD2. Therefore, the corresponding cross-correlation function yields $g_{k \neq l}^{(2)}(\tau)=1$ at all delay times as confirmed by the measurement shown in Fig. 1(c). Coupling of two QDs would be characterized by measuring a deviation from 1 around $\tau=0$ for $g_{k \neq l}^{(2)}(\tau)$.

As an initial characterization, pump-power dependent spectra were taken for 20 individual pairs, all showing similar properties. A schematic of the stacked QDs and corresponding band diagram is given in Fig. 2(a). Under nonresonant laser excitation carriers are generated in the GaAs matrix that relax into the wetting layers and from there into the QDs. Subsequent radiative recombination leads to five dominant emission lines in the power dependent spectra labeled by $X 1-X 5$ [Fig. 2(b)]. At low pump powers there are two dominant peaks $X 1$ and $X 2$ split by energy $\Delta E_{0}$ that varies from 0.5 to $5.0 \mathrm{meV}$ for different QD pairs. As the pump power is increased, the peak intensities increase linearly as expected for single exciton recombination. The spectral lines $X 3, X 4$, and $X 5$ emerge
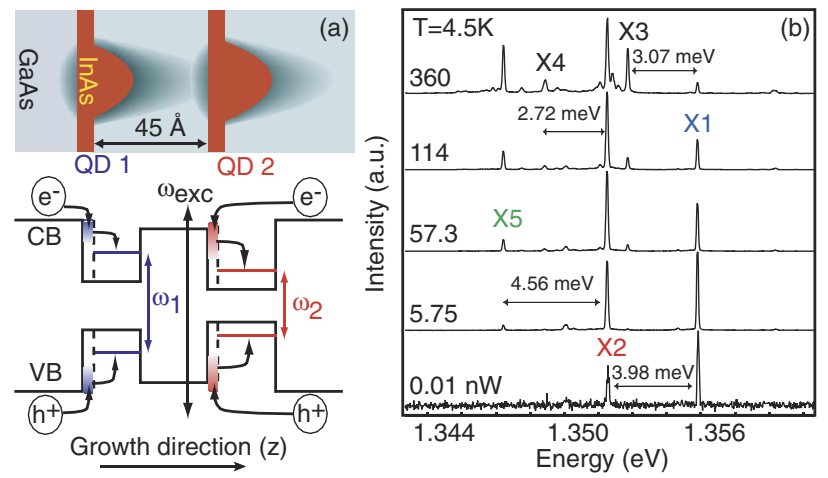

FIG. 2 (color online). (a) Schematic of the sample (top): two layers of InAs/GaAs QDs are vertically stacked with a separation of $45 \AA$. Schematic band diagram (bottom) of the conduction band (CB) and valence band (VB) for two stacked QDs with different transition frequencies $\omega 1$ and $\omega 2$. The pump laser, with energy $\hbar \omega_{\text {exc }}$, generates carriers in the GaAs matrix that relax into the wetting layers and from there into the QDs. (b) Typical power dependent PL spectra from an individual QD pair showing five dominant emission lines, labeled $X 1-X 5$. with quadratic power dependence indicative of biexcitons, while the $X 1$ intensity diminishes. The statistics on 20 individual QD pairs with a separation of $45 \AA$ demonstrates that the upper limit for the coupling energy $\Delta E_{0}$ is quite small, i.e., $\leq 2.6 \mathrm{meV}$ on average. These small splitting energies are consistent with very recent experiments using magnetic [10] and electrical field [11] tuning, revealing anticrossing energies of 1-2 meV. Since both coupling mechanisms, electronic tunneling and dipole-dipole interaction [15], will cause an energy splitting as a function of detuning, it is not clear from such "anticrossing experiments" which mechanism will dominate the coupling.

To get insight into the coupling mechanism, we studied the temperature dependence of the peak intensities of $X 1$ and $X 2$ transition (Fig. 3). With increasing temperature, the $X 1$ intensity decreases while the $X 2$ intensity increases in such a way that the combined intensity remains constant. In addition, the measured lifetimes at $4 \mathrm{~K}$ of the $X 1$ and $X 2$ states determined from autocorrelation measurements are 1.0 and $2.5 \mathrm{~ns}$, respectively. Both observations are indicative for a directional energy transfer from QD1 to QD2 $[7,14]$. This directionality excludes a direct coupling between the two levels. Coupling via the continuous wetting layer states that are $\geq 100 \mathrm{meV}$ away cannot reproduce the observed strong temperature dependence. Instead, a model that couples the $X 1$ and $X 2$ states through a third level, that is, $\sim 10 \mathrm{meV}$ higher in energy than $X 1$, can be fitted to the data (solid line in Fig. 3). The model takes into account the decay rate of the QDs and uses temperature dependent absorption and emission rates for acoustic phonons in thermal equilibrium to couple the levels. Selfassembled QDs are associated with extended wetting layer states that lead to a quasicontinuous absorption background. Depending on the QD confinement potential those extended states can approach the $s$-shell transition energies

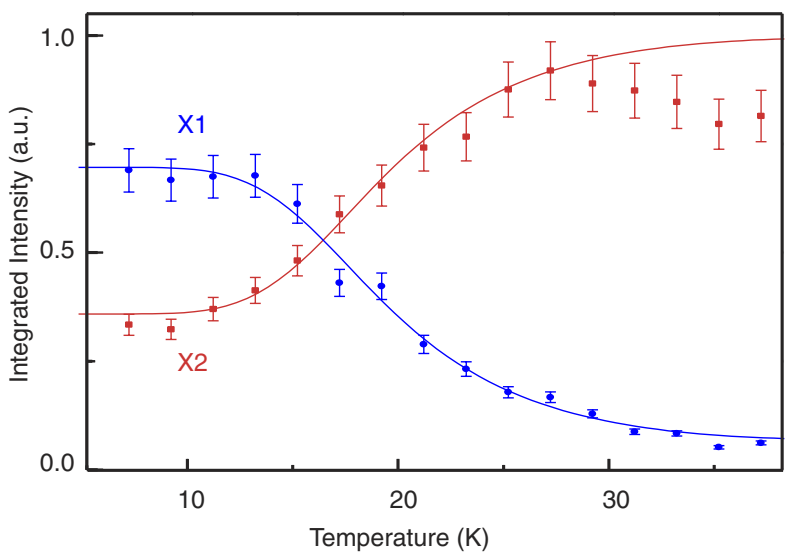

FIG. 3 (color online). PL for a pump power of $1.8 \mathrm{nW}$, of the $X 1$ and $X 2$ transitions versus temperature for a QD pair with $\Delta E_{0}=1.3 \mathrm{meV}$. As the temperature is increased, the intensity from the energetically higher line, $X 1$, is transferred to the $X 2$ line. 
[20]. Evidence that these states are, indeed, important in our InAs QDs has recently been reported [21]. Therefore, absorption of thermal energy (acoustic phonons) can bring the exciton from QD1 into resonance with an extended state of QD2 [14]. From there, the excitation will quickly relax into the $s$-shell leading to emission of the $X 2$ line and thus to a directional energy transfer.

To unambiguously demonstrate that two QDs are coupled, the photon correlations between each spectral line were studied. Autocorrelation measurements on individual spectral lines $(X 1-X 5)$ all show strong antibunching as expected. The main experimental results have been obtained by cross-correlation measurements between the $X 1-X 2, X 1-X 5$, and $X 2-X 5$ lines, shown in Figs. 4(a)4(c). Each cross correlation deviates strongly from $g^{(2)}(0)=1.0$, directly proving that the two QDs form a coupled system. Below a model is proposed that provides an explanation for the observed correlations and spectral signatures.

We identify the $X 1(X 2)$ line as emission from the state with one exciton localized on QD1(QD2). Note that this does include the possibility that only the hole stays localized and the electron wave function is spread over the double dot structure [16]. The notation of $|10\rangle$ and $|01\rangle$ is introduced for these states, where the two indices denote the number of excitons present in each QD. The states $X 3$ and $X 4$ emerge with quadratic power dependence at energies $\sim 3$ meV less than $|10\rangle$ and $|01\rangle$, respectively. As this is the typical Coulomb binding energy for biexcitons in single InAs/GaAs QDs, these states are labeled $|20\rangle$ and $|02\rangle$. In support of this assignment, measurements (not shown) of $g_{|20\rangle,|10\rangle}^{(2)}(\tau)$ and $g_{|02\rangle,|01\rangle}^{(2)}(\tau)$ exhibit the strong cascaded emission expected for biexciton to exciton states [22]. Finally, a new biexciton line $X 5$ emerges in the spectra at lowest photon energy. We assign this to the $|11\rangle$ state with two excitons, one localized on each QD. This state occurs at lowest energy due to an attractive interdot Coulomb interaction that can be larger than the binding energy of the $|02\rangle$ and $|20\rangle$ intradot biexciton states.

To model the measured photon correlations, a four-level rate equation is used that includes a ground state $|00\rangle$, two single exciton states $|10\rangle$ and $|01\rangle$, and an interdot biexciton state $|11\rangle$. The decay rate of the single exciton transitions $\Gamma_{X}$ is assumed to be equal for both dots, and directional energy transfer from $|10\rangle$ to $|01\rangle$ with a rate $W_{T}$ is included. The pump, included via the rate $W_{P}$, induces transitions from the ground state into the $|10\rangle$ or $|01\rangle$ states and from there into the $|11\rangle$ biexciton state. The rate equations for the system are $\partial \vec{n} / \partial t=\mathbf{M} \cdot \vec{n}$, where the matrix $\mathbf{M}$ is

$$
\mathbf{M}=\left(\begin{array}{cccc}
-2 W_{P} & \Gamma_{X} & \Gamma_{X} & \mathbf{0} \\
W_{P} & -W_{P}-\Gamma_{X} & W_{T} & \Gamma_{X} \\
W_{P} & 0 & -W_{P}-\Gamma_{X}-W_{T} & \Gamma_{X} \\
0 & W_{P} & W_{P} & -2 \Gamma_{X}
\end{array}\right) .
$$

The column vector $\vec{n}\left(n_{|00\rangle}, n_{|01\rangle}, n_{|10\rangle}, n_{|11\rangle}\right)$ corresponds to the expectation value to be in a particular state at time $t$.
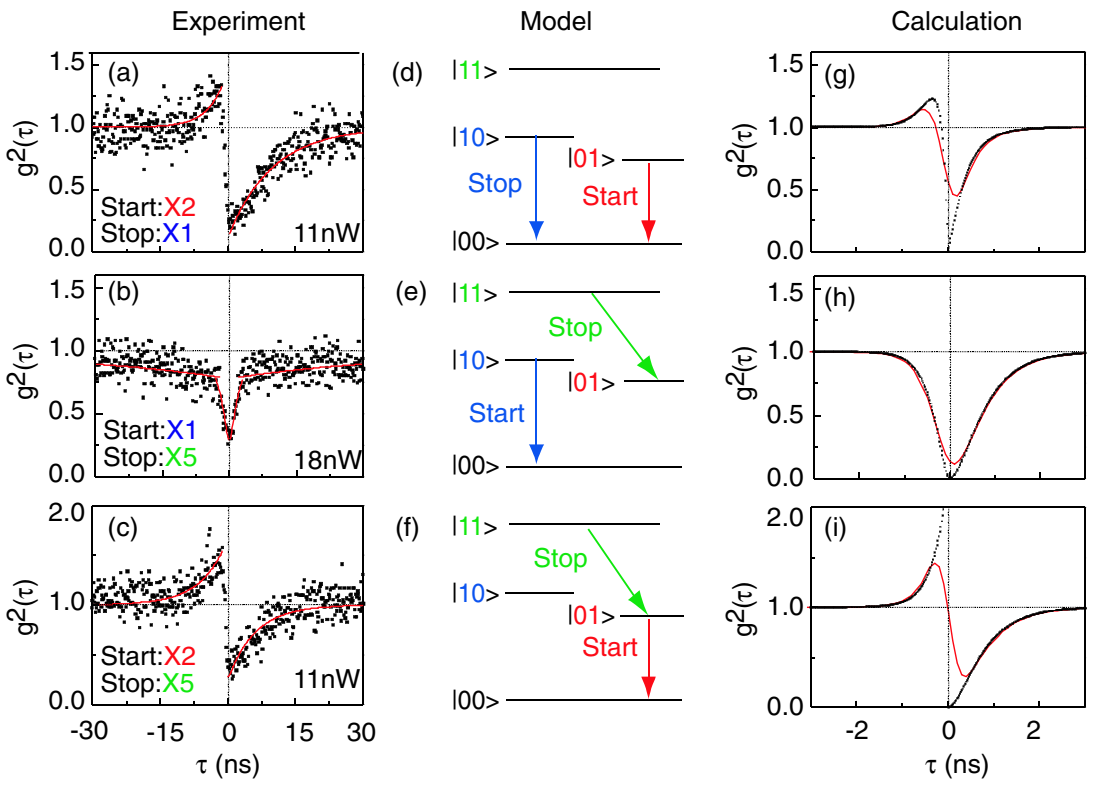

FIG. 4 (color online). Measured cross-correlation functions between the $X 2-X 1, X 1-X 5$, and $X 2-X 5$ lines (a)-(c) compared to the calculated functions between the $|01\rangle$ and $|10\rangle,|10\rangle$ and $|11\rangle$, and $|01\rangle$ and $|11\rangle$ states (g)-(i). All measurements show strong deviations from the uncoupled case $g^{(2)}(\tau)=1$ [see Fig. 1(c)], with minimum values at zero delay time of 0.16 (a), 0.32 (b), and 0.25 (c). Figures 4(d)-4(f) show a level scheme of the states as used in the model, where $|00\rangle$ corresponds to the ground state, $|01\rangle$ and $|10\rangle$ to the single exciton state, and $|11\rangle$ to the interdot biexciton state. The calculated correlation functions (dots) were convolved with a Gaussian curve that reflects the $\sim 700$ ps time resolution of the experimental system (lines). 
Coherences induced through the pump field are neglected as the pump field is far off resonance. The corresponding correlation functions for the parameters $\Gamma_{X}=2 \mathrm{~ns}, W_{P}=$ $0.75 \mathrm{~ns}^{-1}$, and $W_{T}=5.25 \mathrm{~ns}^{-1}$ are shown in Figs. 4(g)4(i) and qualitatively agree with the experimental measurements. In addition, the value for the energy transfer rate is in good agreement with recent calculations assuming a phonon-assisted Coulomb transfer for two nonidentical QDs with 4-5 nm vertical separation, 2-3 meV energy separation, and a lattice temperature of $4 \mathrm{~K}$ [14]. The crosscorrelation results are interpreted as follows: emission of the $X 1$ or $X 2$ line projects the system from the $|01\rangle$ or $|10\rangle$ to the ground state at $\tau=0$. The $X 1$ to $X 2$ cross correlation in Fig. 4(a) is then given by the repopulation of the $|01\rangle$ or $|10\rangle$ state $[14,23]$ and shows antibunching. The cross correlations in Figs. 4(b) and 4(c) involve the $X 5$ emission from the $|11\rangle$ interdot biexciton state into the $|01\rangle$ state. After spectral postselection of the $X 5$ emission the system is never in the $|10\rangle$ state and the $X 1 / X 5(|10\rangle /|11\rangle)$ cross correlation [Fig. 4(b)] shows pronounced antibunching. Conversely, cascaded emission is observed for the $X 2 / X 5(|01\rangle /|11\rangle)$ cross correlation in Fig. 4(c), similar to the quantum cascade of a biexciton to single exciton in single QDs [22]. Note that the model predicts, in addition, a transition from the $|11\rangle$ to the $|10\rangle$ state; however, positive identification of this transition was hindered by the lack of intensity required for the crosscorrelation measurement. While this model qualitatively explains the experimental results, it does not provide an explanation for the long recovery times of $\sim 6 \mathrm{~ns}$ in Figs. 4(a) and 4(c). A possible explanation is the presence of additional metastable states. These can be charged or dark excitons or can be formed via tunneling of the electron only, which is not distinguishable in the current experiment.

We have studied the coupling between two closely stacked self-assembled QDs with carefully tuned $s$-shell transitions to nearly identical energies. The results support the prediction that exciton interdot tunneling is largely suppressed due to the broken symmetry of nonidentical QDs [16], and that the QD coupling is instead dominated by dipole-dipole interactions [14]. In particular, we found a direct Coulomb interaction between the permanent excitonic dipole moments (interdot biexciton), and a directional energy transfer between the QDs, even at their smallest vertical separation of $45 \AA$.

We acknowledge S. Anders, A. Imamoglu, R. Liu, L. Sham, J. Urayama, S. Yaida, and P. Zoller for fruitful discussions. S. Strauf acknowledges support from the Max-Kade Foundation. This research has been supported by DARPA Grant No. MDA972-01-1-0027, NSF NIRT Grant No. 0304678, and AFOSR Grant No. F49620-98$1-0367$.
*Corresponding author.

Electronic address: strauf@physics.ucsb.edu

${ }^{\dagger}$ Present address: University of Cambridge, Cambridge CB3 0WA, U.K.

[1] D. Loss and D. P. DiVincenzo, Phys. Rev. A 57, 120 (1998); A. Imamoglu et al., Phys. Rev. Lett. 83, 4204 (1999).

[2] M. Bayer et al., Nature (London) 405, 923 (2000); R. J. Warburton et al., Nature (London) 405, 926 (2000).

[3] P. Michler et al., Nature (London) 406, 968 (2000).

[4] T. H. Stievater et al., Phys. Rev. Lett. 87, 133603 (2001).

[5] G. Burkard, D. Loss, and D. P. DiVincenzo, Phys. Rev. B 59, 2070 (1999); T. Calarco, A. Datta, P. Fedichev, E. Pazy, and P. Zoller, Phys. Rev. A 68, 012310 (2003); O. Gywat, G. Burkard, and D. Loss, Phys. Rev. B 65, 205329 (2002).

[6] B. W. Lovett, J.H. Reina, A. Nazir, and G. A. D. Briggs, Phys. Rev. B 68, 205319 (2003).

[7] C. R. Kagan, C. B. Murray, and M.G. Bawendi, Phys. Rev. B 54, 8633 (1996); M. Ouyang and D.D. Awschalom, Science 301, 1074 (2003); S. A. Crooker, J. A. Hollingsworth, S. Tretiak, and V. I. Klimov, Phys. Rev. Lett. 89, 186802 (2002).

[8] G. Schedelbeck, W. Wegscheider, M. Bichler, and G. Abstreiter, Science 278, 1792 (1997); I. Shtrichman, C. Metzner, B. D. Gerardot, W. V. Schoenfeld, and P. M. Petroff, Phys. Rev. B 65, 081303 (2002).

[9] M. Bayer et al., Science 291, 451 (2001).

[10] G. Ortner et al., Phys. Rev. B 71, 125335 (2005).

[11] H. J. Krenner et al., Phys. Rev. Lett. 94, 057402 (2005); G. Ortner et al., Phys. Rev. Lett. 94, 157401 (2005).

[12] G. Burkard, G. Seelig, and D. Loss, Phys. Rev. B 62, 2581 (2000); F. Troiani, U. Hohenester, and E. Molinari, Phys. Rev. B 65, 161301 (2002).

[13] E. Biolatti, R. I. Iotti, P. Zanardi, and F. Rossi, Phys. Rev. Lett. 85, 5647 (2000).

[14] A. O. Govorov, Phys. Rev. B 71, 155323 (2005).

[15] A. Nazir et al., Phys. Rev. B 71, 045334 (2005).

[16] G. Bester, A. Zunger, and J. Shumway, Phys. Rev. B 71, 075325 (2005).

[17] B.D. Gerardot, I. Shtrichman, D. Hebert, and P.M. Petroff, J. Cryst. Growth 252, 44 (2003).

[18] The InAs islands were partially covered with GaAs and annealed before complete capping to blueshift the QD $s$-shell transition energy to $\sim 920 \mathrm{~nm}$. We used a 10 and $20 \AA$ GaAs partial coverage for the first and second QD layers, respectively, to tune the $s$-shell transitions.

[19] The QDs are embedded in a photonic crystal membrane that enhances the photon collection efficiency. The QDs transitions are far from resonance with the cavity mode.

[20] Y. Toda, O. Moriwaki, M. Nishioka, and Y. Arakawa, Phys. Rev. Lett. 82, 4114 (1999); A. Vasanelli, R. Ferreira, and G. Bastard, Phys. Rev. Lett. 89, 216804 (2002).

[21] B. Urbaszek et al., Phys. Rev. B 69, 035304 (2004); K. Karrai et al., Nature (London) 427, 135 (2004).

[22] E. Moreau et al., Phys. Rev. Lett. 87, 183601 (2001).

[23] A. J. Berglund, A. C. Doherty, and H. Mabuchi, Phys. Rev. Lett. 89, 068101 (2002). 